\title{
Desarrollo de saberes experienciales y escritura de relatos en la formación docente
}

\author{
Laura PAÑAGUA \\ Diego MARTÍN-ALONSO \\ Nieves BLANCO
}

Datos de contacto:

Laura Pañagua

Universidad de Málaga

laurapani76@uma.es

Diego Martín-Alonso

Universidad de Málaga

diegomartin@uma.es

Nieves Blanco

Universidad de Málaga

nblanco@uma.es

Recibido: 24/01/2021

Aceptado: 15/06/2021

\section{RESUMEN}

En este artículo abordamos el valor formativo de la escritura de relatos de aula para el desarrollo de saberes experienciales en docentes en formación. Hemos llevado a cabo este estudio durante tres años junto a seis estudiantes del grado de Educación Primaria en sus asignaturas de prácticas profesionales, centrándonos en la escritura de relatos a partir de sus vivencias en las escuelas. El propósito es conocer de qué manera la escritura de relatos de aula: i) favorece en los estudiantes el conocimiento de sus ideas pedagógicas; ii) promueve la reflexión sobre cuestiones pedagógicas relevantes; iii) posibilita el desarrollo de un pensamiento propio sobre la educación. Metodológicamente se trata de una investigación con modalidad de auto-estudio, realizando observaciones, entrevistas y el análisis de los relatos elaborados por los estudiantes. En los resultados identificamos tres momentos que posibilitan el desarrollo de saberes experienciales: a) narrar la vivencia de un suceso movilizador; b) reflexionar sobre lo vivido; y c) pensar pedagógicamente, tomando en consideración las situaciones narradas. Concluimos observando la potencialidad formativa de escribir relatos de aula, ya que estos ayudan a profundizar narrativamente la educación, permitiendo el desarrollo de un pensamiento reflexivo con capacidad para captar aspectos relevantes de la vida en las aulas.

PALABRAS CLAVE: saberes experienciales; relatos de aula; auto-estudio; fenomenología; prácticas profesionales. 


\title{
Development of experiential knowledge and writing stories of experience in teacher education
}

\begin{abstract}
In this article we study the educational value of writing stories of experience when trying to promote experiential knowledge in teacher education. We have investigated for three years with a group of six primary teacher students when they were in their Practicum subjects. We have focused on writing stories about their lived experiences at school. The purpose of the research is to study how writing stories of experience: i) facilitates that students become more mindful about their pedagogical ideas; ii) promotes reflection on relevant pedagogical issues; iii) makes possible that students develop their own thinking about education. The methodological approach of the research is a self-study. We have recorded the process by observation, interviews and analysis of the stories made by students. In the results we identify three stages that make possible the development of experiential knowledge: a) narrate the experience of an event that seems relevant for them; b) reflection on the experience; c) pedagogical thought, taking into consideration the narrated stories. Finally, we focus on the teaching potentiality of writing stories of experiences, given that they can help to delve narratively into education, in a way that they allow the development of a thoughtful thinking with the capacity of observing relevant aspects of life at school.
\end{abstract}

KEYWORDS: experiential knowledge; stories of experience; self-study; phenomenology; practicum. 


\section{Introducción}

El oficio docente demanda ponerse en juego en primera persona, ir creando un modo de pensar, una posición propia sobre lo que es deseable, lo que es conveniente hacer. No basta con la aplicación de metodologías o técnicas. En palabras de Biesta (2017, p.162), “enseñar no es una cuestión de seguir fórmulas sino que requiere, en definitiva, docentes que puedan hacer juicios situacionales sabios sobre lo que es pedagógicamente deseable". Las respuestas a la pregunta sobre qué hacer no derivan directamente de los hechos, sino que demanda un proceso de deliberación, de decidir de modo tentativo y siempre incierto qué es lo adecuado para cada situación concreta (Martín-Alonso et al., 2021).

¿Cómo orientar la preparación de los futuros enseñantes de modo que contribuya a la construcción de sus saberes experienciales?, ¿cómo hacer para que estos saberes se conviertan en el anclaje que permita a cada quien decidir qué es lo adecuado y deseable en cada situación educativa? Dado el carácter narrativo de la experiencia y los saberes experienciales (Clandinin, 2013; Contreras y Pérez de Lara, 2010), diversos autores (Conle, 2000; Cifali, 2010; Contreras, 2017) señalan el uso de relatos de aula como una posibilidad para responder a estas cuestiones.

Partiendo de estos estudios, el objetivo que nos proponemos es ahondar en las posibilidades formativas de la escritura de relatos de aula en la construcción de saberes experienciales durante la formación inicial de docentes ${ }^{1}$.

Para ello hemos acompañado a un grupo de estudiantes del grado de Educación Primaria durante sus periodos de prácticas (distribuidos en 3 cursos escolares). Durante el prácticum el recurso formativo principal ha sido la escritura de relatos. A través de la observación de los seminarios, entrevistas con los estudiantes y el análisis documental de las tareas elaboradas por ellos, hemos podido ahondar en su experiencia formativa y, en especial, en su relación con la escritura. De esta manera, desde el marco de una investigación de autoestudio (Vanassche y Keltermans, 2015) y un enfoque fenomenológico (Martín-Alonso, 2019; Van Manen, 2003, 2015), en el análisis de los resultados identificamos dos cuestiones esenciales para que esta sea formativa y contribuya el desarrollo de saberes experienciales: i) se debe invitar a ir más allá de lo anecdótico y circunstancial, tratando de ser sensible a la experiencia de los niños y niñas de manera que se puedan llegar a ver historias en los sucesos escolares; y ii) como formadores debemos buscar que los estudiantes de magisterio se apropien de la escritura buscando la propia voz en el relato y poniéndose en relación con lo narrado.

\footnotetext{
1 Trabajo financiado por el Ministerio de Economía y Competitividad (EDU2016-77576-P) y el Ministerio de Educación, Cultura y Deporte del Gobierno de España (FPU17/04219).
} 


\section{Revisión de la literatura}

\section{Los saberes experienciales y el sentido formativo de las prácticas}

Existe una amplia aceptación de que el conocimiento necesario para la enseñanza está vinculado a la práctica, a decidir qué es lo adecuado en cada situación (Biesta, 2017; Contreras, 2010). Se trata de un conocimiento pático (Van Manen, 2004) que depende de la "comprensión sentida" que tiene el docente de las situaciones que vive.

De este modo, como señalan Clandinin y Husu (2017), a la hora de pensar en el conocimiento necesario para el oficio docente, la conexión experiencia-saber resulta de mayor interés que la tradicional relación teoría-práctica, dado que lo que la enseñanza demanda no es tanto aprender algo nuevo (lo que podríamos llamar teoría), cuanto "habitar" el conocimiento, incorporando eso nuevo a nuestra experiencia. La actividad cotidiana de la docencia necesita que se activen -no que se apliquen- una multiplicidad de saberes que el docente encarna (Recalcati, 2016), y que tienen una diversidad de fuentes entre las que es significativa la propia experiencia (tanto en el ámbito familiar como en el escolar), previa a la formación específica para ser docente (Hedges, 2012).

Los saberes docentes son, entonces, saberes incorporados, personalizados y situados, de modo que se puede decir que los profesores actúan en el aula y se ponen en relación con el alumnado desde su propia experiencia vivida. Por ello, señala Tardiff (2004), el profesor no es un sujeto epistémico sino un sujeto experiencial. Sus saberes son fruto de una transacción constante entre lo que se es -incluyendo lo que se conoce, lo que se siente y lo que se espera- y lo que se hace. No se trata, por tanto, de la acumulación de modos de hacer y de resolver cuestiones en la práctica, sino más bien de una disposición, de "un modo de saber que se cultiva y no solo que se comunica" (Contreras, 2013, p.131), que demanda una relación pensante con la realidad, con lo que sucede y lo que nos sucede. No es un saber en abstracto, sino que se mantiene en estrecha conexión con el presente concreto, en una relación viva de reflexión y acción.

Prepararse para ser docente es establecer una relación de diálogo con uno mismo y con los otros sobre el sentido de la profesión, sobre las cuestiones fundamentales del oficio, a partir de lo que vivimos en situaciones concretas. Requiere que se practique un pensar detenido sobre las experiencias que se viven, teniendo oportunidad de activar "la disciplina mental" de reflexionar sobre su experiencia (Mortari, 2012, p.526). En tanto que la disposición reflexiva no es "natural", es necesario que las y los docentes en formación tengan experiencias formativas que expresamente les propongan reflexionar sobre el sentido de lo que hacen y de lo que sucede en las aulas; y que tengan el acompañamiento que les permita analizar la realidad educativa y su intervención en ella, comprendiendo su sentido pedagógico y cuestionando los supuestos dados (Contreras et al., 2019; Sierra y Blanco, 2017). 
En este sentido, y aunque todas las fases de la formación son necesarias para desarrollar el conocimiento experiencial de los futuros docentes, el práticum cobra una relevancia particular, ya que se trata de un periodo en el que experimentar y aprender sobre sí y sobre el oficio docente como consecuencia de permanecer en un contexto profesional real (Zabalza, 2011). Así, en este contexto, la reflexión puede constituirse como una estrategia formativa que les permite derivar saberes de esta experiencia que viven (Saiz y Susinos, 2017).

\section{La escritura en el proceso de formación}

Sardi (2013, p.14) señala que "escribir sobre lo que nos pasa en la práctica es en sí misma una experiencia de formación". Esto es, la escritura puede llegar a constituirse como una mediación para dar forma al pensamiento, para reconstruir lo vivido y comunicar algo de su sentido. Pero no vale cualquier escritura, no sirve la que habitualmente se practica en la escuela y en la universidad, que se centra en la repetición y la reproducción, sin arriesgar sentido propio (Carlino, 2005). Es preciso recuperar su valor epistémico, de modo que permita distanciarse de las propias ideas para así revisarlas y transformarlas (Bombini y Labeur, 2013; Brailovsky y Menchón, 2014; López, 2018).

El tipo de escritura que necesita la formación que aspira a cultivar una relación fructífera entre experiencia y saber es una escritura personal-reflexiva (Contreras, 2013), que involucra a quien escribe para estar presente con la propia subjetividad, poniéndose en juego (Molina et al., 2016; Pañagua et al., 2019). Se trata de escribir en primera persona para dejarse transformar en el proceso de relatar una vivencia (Cifali, 2010); una escritura personal y reflexiva en tanto que se practica como una indagación de sí, que mantiene la presencia de quien escribe, que se siente parte del relato y que le posibilita ir buscando el sentido de lo que ha sucedido y cómo eso le afecta (Cabrera y Fuentes, 2016). En última instancia, con ella "intentamos poner lo vivido en relación con lo que nos hace pensar, lo sentido con el intento de captarlo, lo pasado con lo que nos sentimos llamados a hacer" (Contreras, 2013, 14).

En resumen, la escritura puede llegar a convertirse un recurso mediador que permita mantener una relación reflexiva con la realidad y que busca dar cuenta de lo vivido para abrirse a nuevas preguntas, contribuyendo así al desarrollo de saberes experienciales. Al mismo tiempo se trata de una oportunidad para explorar y practicar un lenguaje sensible, ligado a la vivencia situacional, que a través de la narración permita pensar sobre situaciones concretas y sobre las cualidades pedagógicas que podemos percibir en ellas (Van Manen y Li, 2002).

\section{Los relatos de aula}

El uso de los relatos en la formación del profesorado tiene una larga historia, que se inscribe en enfoques de investigación narrativa y en concepciones de la 
enseñanza que prestan atención a la experiencia y los saberes de los docentes (Conle, 2000; Contreras, 2017).

En el ámbito de la formación permanente, Cifali (2010, p.183) habla de "relatos de experiencias" para referirse a un dispositivo de formación que posibilita "abordar los problemas relacionales, la intersubjetividad y la subjetividad en los oficios de enseñanza y educación". El relato constituye un modo de aprender de la experiencia, construir conocimiento sobre ella y transmitirlo. Por su parte, Richert (2003) denomina "textos experienciales" a las narraciones que elaboran los docentes (en su caso noveles), que tienen como propósito "captar" la experiencia para aprender sobre sí y sobre su trabajo, haciéndoles conscientes del significado de las propias acciones.

El relato de experiencia o registro de observación (Brailovsky y Menchón, 2014) es una forma habitual de demanda a estudiantes durante su periodo de prácticas, que busca que reflejen su propia experiencia aventurándose a expresar ideas propias. En esta línea, Bombini y Labeur (2013) denominan "las dos hojitas" a un relato corto centrado en una escena de la práctica especialmente relevante para quien la narra, y que luego es compartida con el resto de estudiantes. Por su parte, Sardi (2013) denomina "incidentes críticos" a relatos que narran situaciones en las que se ha vivido algo que resulta inesperado y que reta lo conocido.

En resumen, la escritura de relatos en los procesos de formación busca favorecer que se pueda pensar "con" ellos (y no sobre ellos), en relación a lo que narran y a lo que abren. Y así pueden posibilitar cultivar un saber sensible a lo vivo y que nos involucre completamente. Por eso buscan movilizar preguntas más que ofrecer respuestas y, sobre todo, despertar preguntas existenciales, no preguntas retóricas (Contreras et al., 2019; Molina y Gómez, 2016).

¿Cómo hacer para que, en la escritura, lo vivido se acompañe de pensamiento? El modo en que en nuestra investigación hemos planteado los relatos deriva de una concepción de la formación que busca desarrollar saberes profesionales anclados en la experiencia. Por ello, entendemos que los relatos de aula no consisten solo en narrar lo que se ha vivido, sino que buscamos que escribir el relato sea un modo de dar forma a lo vivido, seleccionando aquellos aspectos que consideran relevantes y prestando atención a lo que, en el proceso de pensar y escribir, va apareciendo y que requiere detenerse para continuar haciéndose preguntas (Contreras, 2016).

Finalmente, no hay reglas fijas para la escritura, pero sí indicaciones respecto a qué se esperaría encontrar en un buen relato: a) detalles concretos y abundantes que se refieran no solo a hechos sino a sentimientos, mostrando el impacto de las emociones en la profesión (Cifali, 2010); b) un marco temporal que vincula el pasado y el presente, no como acumulación u ordenación de hechos sino presentando "una síntesis particular que se debe al sentido" (Han, 2018, p.25); c) un autor que se involucra en el relato, que está presente en él, asumiendo su subjetividad exponiéndose y mostrando sus dificultades y preocupándose por las vidas de quienes aparecen en la historia (Bochner, 2000); d) un espacio que se crea 
para que el lector pueda involucrarse en el relato, con capacidad para conmoverle.

\section{Método}

Este estudio se enmarca en una propuesta formativa con la que pretendemos contribuir a que cada estudiante pueda pensarse como docente, conectando con sus propias ideas acerca de lo educativo. Para ello utilizamos -entre otros recursos narrativos- la escritura de relatos de aula. Dentro de un proyecto de investigación más amplio (centrado en la formación docente y el desarrollo de saberes experienciales), a través de la modalidad de auto-estudio (Vanassche y Kelchtermans, 2015) y desde un enfoque fenomenológico (Martín-Alonso, 2019; Van Manen, 2003), pretendemos analizar las propias prácticas formativas para comprenderlas y valorar su repercusión en la formación de docentes.

En concreto, nuestros objetivos se centran en explorar los modos en que la escritura de relatos de aula durante las prácticas de magisterio: i) favorece el conocimiento sobre las propias ideas pedagógicas; ii) promueve la reflexión sobre cuestiones pedagógicas relevantes; iii) posibilita el desarrollo de un pensamiento propio sobre la educación.

Además de la redacción del diario de prácticas, hemos pedido a los estudiantes que compongan pequeños relatos (no más de una página) a partir de situaciones vividas en los centros y que consideren significativas. Debían reconstruir el suceso descriptivamente, deteniéndose a pensar en lo que significa para él o para ella: cómo lo ha vivido, qué preguntas le despierta, qué le hace pensar y sentir. Además, ponemos énfasis en evitar los juicios, deteniendo el impulso de buscar respuestas y soluciones rápidas, porque no se trata de valorar lo sucedido sino de apoyarse en ello para invitar a pensar sobre algo pedagógicamente importante para uno mismo.

Siguiendo esta propuesta, hemos trabajado con un grupo de 6 estudiantes (4 chicas y 2 chicos) del grado de Educación Primaria de la Universidad de Málaga a lo largo de los tres años en que desarrollan sus prácticas. Nuestra relación se ha concretado a través de los seminarios ( 3 en el primer periodo, 4 en el segundo y 5 en el tercero), entendidos como espacios para reflexionar sobre las experiencias vividas en las aulas y sobre la escritura de los relatos. Estos relatos han sido leídos y acompañados a través de una lectura semanal, tras la que compartíamos con cada una/o las sugerencias, ideas y reflexiones que nos suscitaban.

Durante los seminarios (12 en total) hemos realizado observaciones y grabaciones de audio que posteriormente se han transcrito. Al finalizar el último curso, la autora 1 ha mantenido entrevistas con cada estudiante (grabadas y transcritas), abordadas desde una disposición hermenéutica (Van Manen, 2003) y enfocadas a: i) conocer cómo han vivido el proceso de escritura de relatos; ii) el valor que les dan en su formación; iii) la consciencia que tienen sobre sus ideas pedagógicas y su evolución.

Paralelamente, siguiendo los planteamientos propios de la investigación 
educativa-cualitativa (Stake, 2010) hemos realizado el análisis documental (Padgett, 2016) de los relatos de aula que han elaborado los estudiantes (un centenar), buscando descubrir los elementos significativos y la evolución de los mismos a lo largo del tiempo. Siguiendo las orientaciones metodológicas del enfoque fenomenológico (Van Manen, 2003) hemos analizado los datos buscando los aspectos estructurales o temáticos de la experiencia vivida por el alumnado en el proceso de escritura de relatos de aula. Para encontrarlos hemos procedido a realizar una lectura global tratando de captar el significado que cada estudiante atribuye a sus relatos para, posteriormente, realizar una aproximación selectiva, señalando frases y palabras reveladoras de sentido. La discusión de los resultados se elabora, en coherencia con el enfoque fenomenológico desde el que se realiza el análisis, siguiendo la propuesta que Van Manen (2003) denomina "temática”, es decir, utilizando como guías los temas que han surgido en el análisis.

\section{Resultados}

En sus relatos de aula, los estudiantes han abordado diversas cuestiones que les han permitido poner la atención y pensar sobre cuestiones de gran relevancia pedagógica. Los que aparecen con mayor frecuencia son la relación educativa, las relaciones entre niños y niñas en el aula, así como el rol docente, su complejidad y la responsabilidad que implica. Otros temas en los que se han centrado, aunque no con la misma frecuencia, han sido: las necesidades de la infancia y el modo en que son atendidas -o no- en la escuela; la organización del tiempo y los espacios (como la biblioteca o el recreo); la evaluación; y cuestiones metodológicas relacionadas con los materiales y recursos didácticos.

En el análisis de estos relatos y la vivencia de su proceso de escritura hemos observado la presencia de tres momentos que han favorecido el desarrollo del pensamiento pedagógico: i) la narración del suceso; ii) la reflexión sobre la vivencia del mismo; y iii) pensar sobre su sentido pedagógico.

El primer momento que percibimos en los relatos es la narración de la vivencia. Para que un suceso nos mueva es necesario que seamos capaces de "verlo". Por eso, la elección del acontecimiento en el que centrarse suele resultar difícil. En cinco horas de clase pasan muchas cosas, pero no siempre nos interpelan y nos invitan a replantearnos nuestra visión. Casi ningún relato se construyó a partir de un acontecimiento que se identificara previamente como significativo o revelador, sino que éste fue movilizando al estudiante a medida que iba construyendo una relación narrativa con su vivencia. Así, puede decirse que las vivencias que relatan no les plantean preguntas hasta que no han comenzado a narrarlas, por ejemplo, en el diario de prácticas:

Una cosa es que algo te llame la atención, y otra es profundizar más en eso [...] en el momento no te da tiempo a pensar (...) Algunos relatos los escojo cuando voy haciendo el diario y me doy cuenta de algo que es importante; y lo separo para reflexionar luego sobre ello. (María) 
El inicio de construcción de un relato consiste en centrar la mirada en un evento $\mathrm{y}$, para que esto suceda, es necesario escribir sobre el acontecimiento de forma detallada, fijándose en los protagonistas, los sucesos, y nuestra posición respecto a ellos.

Cuando acudí por primera vez con los pequeños a la Biblioteca en el recreo, quedé impresionada. En ella se encontraba una multitud de pequeñas criaturas, algunos buscaban libros, otros hacían sus deberes, otros hacían uso de los ordenadores, etc. Todos en silencio, como si al entrar allí, se sumergiesen en otro mundo. Jamás había visto una biblioteca escolar tan habitada. (Fragmento de relato de Reme)

Narrar es poner palabras a la experiencia, reconstruyendo narrativamente lo que hemos vivido (Martín-Alonso et al., 2019), de manera que la vivencia se transforma en historia. Se trata de una historia que yo he vivido y que, al mismo tiempo, me hace ver historias en las otras personas.

Cuando vas escribiendo, aunque no te des cuenta, vas pensando y te centras más en lo que ha sucedido. A lo mejor ves que una niña está nerviosa y te quedas ahí; pero cuando te pones a escribir te planteas porqué se ha puesto nerviosa. Es como mirar el antes y el después, en lo que ha pasado y porqué ha pasado. (Sara).

El segundo momento del proceso de escritura de relatos es la reflexión sobre lo vivido. Relatar una experiencia vivida en la escuela posibilita iniciar una relación narrativa con el suceso (Clandinin, 2013) y, de este modo, comienzan a surgir inquietudes y preguntas sobre ellas y sobre la propia historia del estudiante en relación con la vivencia narrada. Así aparecen preguntas sobre lo que esa realidad despierta, lo que dice y qué sentido tiene, estableciéndose conexiones entre la persona, lo que sucede y la historia de los protagonistas.

Me gustaría intentar dar respuesta a la pregunta que Fernando [un alumno de la escuela] me lanzó durante nuestra conversación: “¿cómo te sentirías si todo el día te dijeran todo lo que haces mal?” Esto me hace pensar que, al igual que Fernando, yo necesito ver que alguien confía en mí, que por mucho que le falle a alguien me da ánimos para seguir y hacerlo bien. (Fragmento de relato de Marta)

En consecuencia, comienzan a surgir preguntas sobre lo que esa realidad nos despierta, qué nos dice y qué sentido tiene. En los relatos, la reflexión tiene un sentido narrativo, poniendo en relación la historia relatada con la propia, de modo que se produce una "resonancia", una conexión entre lo que ha sucedido y lo que se ha vivido en primera persona (Conle, 2003). Así, al reflexionar, aquello que ha sucedido vuelve a cobrar vida, pero ahora a través de uno mismo, de lo que piensa, de lo que es. A la vez que se hace propia la realidad, se establecen conexiones entre la persona, lo que sucede y la historia de los protagonistas, buscando un sentido pedagógico.

Recuerdo que cuando era niña me encantaba dibujar y colorear. Hoy lo detesto. Me han exigido tanto en esta tarea cuando era pequeña, han 
limitado tanto mi potencial creativo, que poco a poco fui abandonando esta actividad [...] La reprimenda que Juan recibió por parte de Amanda pudo ayudar a incrementar su miedo a equivocarse. Si no estás abierto a equivocarte, nunca se te va a ocurrir algo original. ¿Cómo podemos desarrollar la creatividad de los niños y niñas sin limitarles de acuerdo a unos patrones fijados? (Fragmento de relato de María)

En esta línea, hemos observado que los relatos en los que los estudiantes se hacían preguntas pedagógicas de mayor complejidad están escritos en primera persona. Dicho de otra manera, para que tenga lugar la reflexión sobre el sentido pedagógico de lo vivido es necesaria una disposición a "dejarse tocar" por la experiencia (Molina et al., 2016). Sin embargo, cuando no ha existido esta disposición a conectar la vivencia con uno mismo (algo que se ha manifestado con una escritura impersonal o en tercera persona), aparece el "rictus reflexivo" (Brailovsky y Menchón, 2014) que redunda en lugares comunes en vez de plantearse preguntas auténticas. Algo que observamos de forma paradigmática en uno de los relatos de Juan:

Cuando un niño tiene ciertas actitudes inadecuadas de manera reiterativa, los profesores suelen etiquetarlos como niños "malos"; pero... ¿realmente lo son? Los docentes tendremos que tratar con alumnos igualmente distintos y nuestro deber es intentar que sean buenas personas y que entiendan que toda acción tiene su consecuencia, sea buena o mala.

De este modo, cuando el alumnado se ha distanciado de la experiencia, más que abrir interrogantes y movilizar, la escritura de relatos ha llevado a buscar respuestas rápidas y soluciones cerradas. Esta posición ha dificultado la construcción de saberes pedagógicos, ya que para ello es necesaria una reflexión que incite a lo nuevo, a un replanteamiento de los saberes propios. En definitiva, la experiencia ha movilizado al alumnado cuando este se ha puesto en relación con la propia historia, haciendo que el estudiante piense y se cuestione sobre sí mismo y sus saberes:

Estoy segura que esta no ha sido una tarea fácil para Laura (la maestra). De repente te encuentras con un alumno de 6 años que te insulta, provoca numerosas disputas con sus compañeros, golpea objetos contra las paredes, etc. Pero, ¿qué hizo Laura para que el comportamiento de Ramón fuese cambiando? ¿Afecta a un docente la situación familiar de un alumno? ¿Qué habilidades debe tener un profesor para atender a un alumno con este tipo de circunstancias? ¿Hasta qué punto somos los docentes importantes en la vida de un niño? ¿Somos realmente conscientes de ello? (María).

El tercer y último momento en la escritura de los relatos es pensar pedagógicamente, algo que surge al ponerse en relación con la vivencia de un modo reflexivo, pensando sobre sí y sobre la pedagogía. Este es el propósito de la narración, la transformación propia a partir de una mayor consciencia del sentido que nos orienta. Esta nueva conexión con la vivencia se incluye en la propia 
historia, transformándola (Contreras, 2017).

En los relatos, este tránsito se manifiesta en las proyecciones que como docentes hacen acerca de sí mismos y en nuevos pensamientos acerca de cuestiones de naturaleza pedagógica (el rol docente, la infancia, las relaciones, la evaluación, etc.), también cuando asumen un compromiso y definen su propio perfil profesional. Por ejemplo, María pudo pensar sobre los exámenes y las calificaciones, que se han convertido en un aspecto significativo de su modo de entender la profesión docente:

¿Por qué usar el término "examen" para saber lo que ha aprendido o no un alumno? ¿Es necesario? ¿Por qué se le da tanta importancia? ¿Cómo afectan este tipo de pruebas a niños tan pequeños? Estas son algunas de las preguntas que me han surgido al ver situaciones como estas que he relatado... La angustia que provoca a las criaturas, como a Gina, no solo puede tener efectos en el presente sino producir consecuencias negativas en el futuro. Seguramente muchos docentes piensan que se trata de una buena manera de verificar el aprendizaje del alumnado pero, si nos paramos a pensar, ¿no podríamos hacerlo de otras formas más efectivas y positivas?

En cambio, Marta ha centrado su atención en el alumnado, construyendo significados en torno al rol docente, a la infancia y a la relación pedagógica entre ambos.

¿No se dice que lo más importante de las relaciones es la confianza? La relación alumno-maestro puede ser que también funcione así, el maestro necesita confianza por parte del alumno de que lo va a saber guiar de una manera adecuada. El niño necesita la confianza del maestro para contar con él, lo que nos facilitará la labor como maestros. Depositar confianza. Un día en mí la depositaron y hoy estoy aquí, escribiendo estas líneas para intentar llegar a donde quiero llegar.

\section{Discusión}

En esta propuesta formativa planteamos la escritura de relatos de aula como mediación en el desarrollo de los saberes necesarios para el oficio docente, posibilitando conectar en cada estudiante las vivencias en las escuelas con sus propias historias, inquietudes y necesidades. Al examinar los resultados podemos apreciar cómo la escritura de relatos ha permitido a los estudiantes pensar en el sentido de lo vivido, haciéndose más conscientes de que hay un paso adicional a la vivencia, la reflexión, que posibilita prestar atención a aspectos estructurales de la relación educativa. Para que esto sea posible hemos identificado dos temas esenciales del proceso de escritura de relatos y su acompañamiento: i) trascender lo anecdótico, siendo sensible a la experiencia de los niños y niñas de manera que se puedan llegar a ver historias (sus vidas) en los sucesos escolares; y ii) apropiarse de la escritura buscando la propia voz en el relato y poniéndose en relación con lo narrado. 


\section{Ver historias en los sucesos}

En el contexto escolar siempre están sucediendo acontecimientos y se están viviendo historias. Pero hay que saber verlas y componerlas. Es decir, hemos de captar el sentido de un suceso, y pensar sobre él para poder crear y re-crear la historia que acompaña a esos hechos, que los sitúa en una trama, en la vida de alguien (Contreras, 2016a). Por ello el primer reto formativo que se nos ha planteado ha sido ayudar a los estudiantes a "ver" los relatos en la vida del aula. Es necesario, en definitiva, pensar la enseñanza y el currículum como vida que se vive (Martín-Alonso et al., 2019).

En este sentido, aunque los relatos suelen ser pequeñas "escenas", exigen "tirar del hilo" que están mostrando, de manera que nos permiten profundizar narrativamente la educación, buscando conectar una situación cotidiana con el sentido profundo que subyace en ella (Contreras et al., 2019).

Para ello, como hemos visto en los tres momentos de la composición de relatos, la escritura necesita tiempo y sosiego, pensar en una situación o vivencia concreta, repensar su sentido, buscar ir más allá de lo evidente, y también permitir que nos afecte, que nos deje alguna huella. Pero también exige centrar la mirada, poner la atención en aspectos o cualidades concretas y, de ese modo, examinarlas como nuevas, yendo más allá de lo ya conocido.

Por tanto, para que el alumnado haya podido descubrir la esencia de una experiencia ha debido detenerse, concentrarse, dedicarle tiempo. El necesario, ni acelerado ni dilatado, porque "la dilación y las prisas son dos de los síntomas de la falta de tensión narrativa" (Han, 2018, p.47). Así, la escritura de relatos exige una cierta lentitud para que los sucesos puedan condensarse y cristalizar en historias, algo que hay que hacer coexistir con la compresión de los tiempos académicos.

Por último, se ha revelado como importante el intercambio de relatos con las compañeras y compañeros. Como señaló Richert (2003), al ser al mismo tiempo lectores de los relatos de los demás y autores de los propios, se puede acceder a una variedad de situaciones y de modos de vivirlas y entenderlas que amplían las perspectivas propias y tienen capacidad para acercarse a la complejidad y multidimensionalidad de la enseñanza.

\section{Apropiarse de la escritura}

Aunque deben cuidarse los aspectos formales de la escritura, no hay que olvidar que es un vehículo para expresar pensamiento y que lo central es el sentido de los relatos, el propósito para el que los componemos. Por tanto, la forma de narrar, siendo importante, no es central puesto que el relato no es un modo de contar más atractivo, más sugerente o con más capacidad de captar la atención. Lo relevante es en qué medida un relato nos permite mostrar la experiencia educativa tal como la viven docentes, estudiantes, o uno mismo; y en qué medida esa narración nos ayuda a dar cuenta de cualidades sustantivas de lo educativo, nos 
permiten mostrar lo que es difícilmente visible. En definitiva, lo sustancial es cómo hacemos que un relato sea concebido pedagógicamente para que ayude a pensar tanto a quien escribe como a quien lee.

En consecuencia, es preciso cuidar que los relatos sean auténticos. Algo que tiene que ver con la demanda que hace Cifali (2010) de involucrarnos en la escritura, de ser sinceros, expresando lo que sabemos y sentimos, también nuestras limitaciones y dificultades. Autenticidad también en tanto la escritura nos permite expresar nuestra visión sobre lo sucedido relatando nuestras vivencias en un lenguaje propio, no disciplinar. Además, en el contexto de la formación inicial, es preciso alejar el riesgo de escribir tratando de cumplir con las expectativas del profesor. Sabiendo que estamos ante la paradoja de que los estudiantes escriben para responder a una demanda externa que buscamos que sea vivida como propia (Sierra et al., 2017).

Lo que hemos podido constatar en nuestro estudio es la complejidad de esta apropiación. Es necesario cuidar que escriban sin presión, con el apoyo de un lector-crítico (Brailovsky y Menchón, 2014) que no juzga, sino que entra en relación con el texto dejándose sorprender y dialogando con lo que sugiere para plantearse preguntas, para abrir posibilidades y reflexiones; una lectura que lejos de marcar un cierre suponga un inicio, una invitación a continuar explorando.

A pesar de las dificultades, hemos podido evidenciar que, en este proceso de escribir relatos de aula, los docentes en formación tienen la oportunidad de desarrollar un pensamiento propio vinculado a su experiencia. Al escribir, "se fluidifican y descongelan los sentidos petrificados de las palabras [...] nos habremos vuelto más atentos hacia lo particular, nos habremos alejado de las creencias comunes" (Larrauri, 2001, p.23). De este modo creamos una distancia respecto a lo ya conocido, necesaria para que algo nuevo aparezca. Y ahí hay una oportunidad de relacionarse de un modo más reflexivo, con más comprensión, con las propias vivencias e ideas, con el sentido atribuido a los hechos, acercándonos con consciencia a su naturaleza, a sus orígenes, a las tensiones entre lo que hacemos y lo que deseamos, a nuestros prejuicios, a lo que no cuestionamos (Contreras, 2016b). Al poner palabra a lo que vivimos nos hacemos conscientes de ello y nos permite acercarnos más al modo en que comprendemos la educación y a los sentidos que nos orientan en la relación con ella.

\section{Conclusión}

En educación las situaciones son imprevisibles, singulares, y en tanto no hay respuestas previas nos requieren actuar reflexivamente. La reflexión se constituye así en un ingrediente imprescindible en el oficio docente (Korthagen, 2010) y, por tanto, en la formación del profesorado (Mortari, 2012). Esta es una reflexión que debe ayudarnos a confiar en nuestro propio juicio, a partir de nuestra experiencia, y supone "un aprendizaje interior" porque nos permite "una movilización constante de los saberes que ella nos autoriza" (Cifali, 2010, p.182). Un 
aprendizaje interior que nos posibilita plantearnos preguntas sin esperar una respuesta inmediata y cierta, y que nos permite acoger la incertidumbre.

¿Cómo sostener esta reflexión y este aprendizaje interior? ¿Puede la escritura mediar en la construcción de saberes que acojan la incertidumbre del oficio docente y la relación educativa? Movidos por estas preguntas, a lo largo de tres años hemos acompañado a un grupo de estudiantes en sus prácticas, promoviendo una escritura reflexiva capaz de generar preguntas potentes y con sensibilidad para captar aspectos relevantes de la educación. A lo largo de este periodo hemos ido viendo cómo los estudiantes: i) han ido centrando la atención en situaciones potencialmente relevantes, siendo sensibles a las criaturas y sus tribulaciones; ii) han aprendido a seleccionar situaciones de interés, con potencial de análisis y que permiten pensar sobre cualidades relevantes de lo educativo; iii) y han ido reescribiendo, tirando de hilos que les hemos sugerido y que han hecho propios, poniéndose en relación con las escenas que relataban. Todo esto nos sugiere que se han ido haciendo conscientes del modo en que la escritura de relatos, y el proceso de pensamiento que la acompaña, contribuye a su proceso de formación.

Concluyendo, trabajar con relatos en la formación inicial docente tiene un enorme potencial y plantea retos que nos exigen estar alerta para sostener el sentido con el que los llevamos al aula. La escritura de relatos es valiosa pero también compleja, pues requiere que, como docentes, realicemos un acompañamiento cuidadoso y exigente si pretendemos que tengan potencial formativo. Cifali (2010, p.181) nos recuerda que es importante la presencia de alguien que "nos ayuda a mirar lo que no queremos ver, a comprender -por ejemplo cuando tenemos miedo- cómo se nos escapan muchos signos". Y de este modo es como podemos aprender a nombrar, a poner en palabras nuestra experiencia. Un acompañamiento que ayude a ser consciente de las teorías pedagógicas que nos guían, de lo que orienta nuestros modos de interpretar, de mirar, de hacer.

\section{Referencias}

Biesta, G. (2017). El bello riesgo de educar. SM.

Bochner, A. (2000). Criteria against ourselves. Qualitative Inquiry, 6(2), 266272.http://dx.doi.org/10.1177/107780040100700201

Bombini, G. y Labeur, P. (2013). Escritura en la formación docente: los géneros de la práctica. Enunciación, 18(1), 19-29.

Brailovsky, D. y Menchón, A. (2014). Estrategias de escritura en la formación. La experiencia de enseñar escribiendo. Noveduc.

Cabrera, V., y Fuentes, L. (2016). Diarios narrativos: relaciones y encuentros en educación secundaria. Qualitative Research in Education, 5(3), 309-331. http://dx.doi.org/10.17583/qre.2016.1934

Carlino, P. (2005). Escribir, leer, y aprender en la universidad. Una introducción 
a la alfabetización académica. FCE.

Cifali, M. (2010). Enfoque clínico, formación y escritura. En L. Paquay, L. (Coord.), La formación profesional del maestro. Estrategias y competencias (pp. 170-196). FCE.

Clandinin, D. J. (2013). Engaging in narrative inquiry. Left Coast Press.

Clandinin, D. J., y Husu, J. (Eds.). (2017). The Sage handbook of research on teacher education. SAGE.

Conle, C. (2000). Narrative inquiry: Research tool and medium for professional development. European Journal of Teacher Education, 23(1), 49-63. http://dx.doi.org/10.1080/713667262

Conle, C. (2003). An anatomy of narrative currícula. Educational Researcher, $32(2), 3-15$.

Contreras, J. (2010). Ser y saber en la formación didáctica del profesorado: una visión personal. Revista interuniversitaria de formación del profesorado, $68(24,2), 61-82$.

Contreras, J. (2013). Experiencia, escritura y deliberación: Explorando caminos de libertad en la formación didáctica del profesorado. Inter-Ação, 38(1), 1-35.

Contreras, J. (2016a). Relatos de experiencia, en busca de un saber pedagógico. Revista Brasileira de Pesquisa (Auto)Biográfica, 1(1), 14-30.

Contreras, J. (Coord.). (2016b). Tensiones fructíferas: explorando el saber pedagógico en la formación del profesorado: una mirada desde la experiencia. Octaedro.

Contreras, J. (Coord.). (2017). Enseñar tejiendo relaciones. Una aproximación narrativa a docentes y sus clases de educación infantil y primaria. Morata.

Contreras, J. y Pérez de Lara, N. (2010). Investigar la experiencia educativa. Morata.

Contreras, J., Quiles, E. y Paredes, A. (2019). Una pedagogía narrativa para la formación del profesorado. Márgenes, Revista de Educación de la $\begin{array}{llll}\text { Universidad de } & \text { Málaga, } & \text { 58), }\end{array}$ http://dx.doi.org/10.24310/mgnmar.v0i0.6624

Han, B-C. (2018). El aroma del tiempo. Un ensayo filosófico sobre el arte de demorarse. Herder.

Hedges, H. (2012). Teachers funds of knowledge: a challenge to evidencebased practice. Teachers and Teaching: theory and practice, 18(1), 7-24.

Korthagen, F. (2010). La práctica, la teoría y la persona en la formación del profesorado. Revista Interuniversitaria de Formación del Profesorado, 68(24,2), 83-101.

Larrauri, M. (2001). La libertad según Hannah Arendt. Tándem. 
Molina, M.D., y Gómez, D. (2016). Señales de vida en la universidad. Cuadernos de Pedagogía, 470, 68-70.

López Secanell, I. (2018). Ser en Proyecto: una autoetnografía del proceso de construcción del proyecto vital de una profesora de Educación Física. Qualitative Research in Education, 7(1), 36-63. http://dx.doi.org/10.17583/qre.2018.3051

Martín-Alonso, D. (2019). El tejido curricular. Indagación narrativa sobre la relación educativa y el proceso de creación curricular [Universidad de Málaga]. https://hdl.handle.net/10630/19311

Martín-Alonso, D., Blanco, N., \& Sierra, J. E. (2019). El proceso de creación curricular en estudiantes de educación secundaria. Una indagación narrativa. Profesorado, Revista de curriculum y formación de profesorado, 23(2), 377395. https://doi.org/10.30827/profesorado.v23i2.9692

Martín-Alonso, D., Blanco, N., \& Sierra, J. E. (2021). Estudio fenomenológico sobre las disposiciones docentes en la relación pedagógica. Magis, Revista Internacional De Investigación En Educación, 14, 1-23.

Molina, M. D., Blanco, N., \& Arbiol, C. (2016). Dejarse tocar para que algo nos suceda. En J. Contreras (Ed.), Tensiones fructíferas: Explorando el saber pedagógico en la formación del profesorado. Una mirada desde la experiencia (pp. 111-150).

Mortari, L. (2012). Learning thoughtful reflection in teacher education. $\begin{array}{lll}\text { Teachers } \quad \text { Teaching, 18(5), } & \text { 1-21. }\end{array}$ http://dx.doi.org/10.1080/13540602.2012.709729

Padgett, D. K. (2016). Qualitative methods in social work research. Sage Publications

Pañagua, L., Martín-Alonso, D., \& Blanco, N. (2019). Escritura reflexiva y desarrollo de saberes experienciales. Tensiones y posibilidades. Revista Interuniversitaria de Formación del Profesorado, 94(33), 11-28.

Stake, R. E. (2010). Investigación con estudio de casos. Morata.

Recalcati, M. (2016). La hora de clase: Por una erótica de la enseñanza. Anagrama.

Richert, A. (2003). La narrativa como texto experiencial: incluirse en el texto. En A. Lieberman y L. Miller (Coords.), La indagación como base de la formación del profesorado y la mejora de la educación (pp. 193-208). Octaedro.

Saiz, A. y Susinos, T. (2018). Problemas pedagógicos para un Practicum reflexivo de Maestros. Revista Complutense de Educación, 28(4), 993-1008. http://dx.doi.org/10.5209/RCED.50924

Sardi, V. (2013). La escritura de las prácticas en la formación docente en Letras. 
En V. Sardi (Coord.), Relatos inesperados. La escritura de incidentes críticos en la formación docente en Letras (pp. 10-32). Universidad de La Plata.

Sierra, J. E., \& Blanco, N. (2017). El aprendizaje de la escucha en la investigación educativa. Qualitative Research in Education, 6(3), 303-326.

Sierra, J. E., Caparrós, E., Molina, M. D., \& Blanco, N. (2017). Aprender a través de la escritura. Los diarios de prácticas y el desarrollo de saberes experienciales. Revista Complutense de Educación, 28(3), 673-688.

Tardiff, M. (2004). Los saberes del docente y su desarrollo profesional. Morata.

Van Manen, M. (2003). Investigación educativa y experiencia vivida. Idea Books.

Van Manen, M. (2004). El tono en la enseñanza: el lenguaje en la pedagogia. Paidós Ibérica.

Van Manen, M. (2015). Pedagogical tact: knowing what to do when you don't know what to do. Left Coast Press.

Van Manen, M. y Li, S. (2002). The pathic principle of pedagogical language. Teaching and Teacher Education,18(2), 215-224. http://dx.doi.org/10.1016/S0742-051X(01)00065-8

Vanassche, E. y Keltermans, G. (2015). The state of the art in self-study of teacher education practices: a systematic literature review. Journal of $\begin{array}{llll}\text { Curriculum Studies, } & \text { 508-528. }\end{array}$ http://dx.doi.org/10.1080/00220272.2014.995712

Zabalza, M.A. (2011) El practicum en la formación universitaria: estado de la cuestión. Revista de Educación, 354, 21-43. 
